\title{
Migration of a Ruptured, Silicone Gel-Filled Breast Implant into Sternal and Abdominal Areas
}

\section{Heon Yoo, Seung Jun Shin, Myong Chul Park}

Department of Plastic and Reconstructive Surgery, Ajou University Hospital, Suwon, Korea
No potential conflict of interest relevant to this article was reported.

\begin{abstract}
A 50-year-old woman visited our hospital for two palpable masses on her sternal and left upper abdominal areas. She had augmentation mammoplasty 12 years prior. Based on the physical examination, the mass in the sternal area was firm, movable, and round. The size of the mass was approximately $3.0 \times 3.0 \times 1.0 \mathrm{~cm}^{3}$. The mass in the upper-left abdomen showed similar characteristics, with a size of approximately $10.0 \times 15.0 \times 1.5$ $\mathrm{cm}^{3}$. Ultrasonography revealed a $3.9 \times 1.0 \times 3.4 \mathrm{~cm}^{3}$ hypoechoic lesion in the sternal area. On enhanced-mode computed tomography, a rupture of the left breast implant was noted. Both masses had similar densities as the implant. After rupture of the breast implant, surgery was performed under general anesthesia. Using an inframammary approach, both breast implants and masses were removed using the site of the previous mammoplasty incision. Intraoperative findings revealed that the left breast implant was ruptured and the masses consisted of implant gel components. After surgery, the patient's recovery was uneventful. The patient was discharged without any problem on hospital day 9.
\end{abstract}

Keywords Breast implant, Complication, Migration, Rupture

\section{INTRODUCTION}

Breast implants have been used for breast reconstruction and augmentation for the last 50 years. There are about 100,000 women in the United Kingdom with silicone implants, of which approximately $60 \%$ are for breast reconstruction following mastectomy [1]. Approximately one to two million women have undergone breast implantation in the United States [2]. The silicone Poly Implant Prothèse breast implant (also marketed as M-Implants and Rofil-implants) were made in France from 2001 until March 2010 [3]. Capsular contracture is a common complication following breast augmentation surgery [4]. In a 25-year prospective, population-based study, capsular contracture and malposition were reported as the

Received: Oct 14, 2014 Revised: Oct 26, 2014 Accepted: Oct 26, 2014

Correspondence: Myong Chul Park Department of Plastic and

Reconstructive Surgery, Ajou University Hospital, 206 Worldcup-ro,

Yeongtong-gu, Suwon 443-721, Korea. E-mail: mpark@ajou.ac.kr

Copyright (@ 2014 The Korean Society for Aesthetic Plastic Surgery.

This is an Open Access article distributed under the terms of the Creative Commons Attribution Non-Commercial License (http://creativecommons.org/licenses/by-nc/3.0/) which permits unrestricted non-commercial use, distribution, and reproduction in any medium, provided the original work is properly cited. www.e-aaps.org first and third most common reasons for secondary surgery, respectively. Rupture has also long been considered one of the key complications of silicone-gel breast implants [5]. Rupture of silicone implants has received increased scrutiny over the years. Implant ruptures can be overt or silent. Sensitivity of plastic surgeons to a diagnosis of rupture is estimated to be approximately $30 \%$. However, the gross migration of the gel from ruptured breast implants associated with gel bleeding into either the breast parenchyma or surrounding areas - is rare [6]. The migration of silicone gels as subcutaneous nodules can cause local reactions such as siliconoma. Here we report a case of a woman with the distant migration of silicone gel from a ruptured breast implant into the sternal and abdominal areas.

\section{CASE REPORT}

The patient is a 50-year-old woman with bilateral augmentation mammoplasty. The patient noticed a mass in her sternal area several years ago. She presented with a form, movable mass in the sternal area. The size of the mass was $3 \mathrm{~cm} \times 3 \mathrm{~cm} \times 1.0 \mathrm{~cm}$ with similarity to lipoma. Another mass was $10 \mathrm{~cm} \times 15 \mathrm{~cm} \times 1.5 \mathrm{~cm}$ in her upper-left abdomen. Both masses were solid with well-defined mar- 


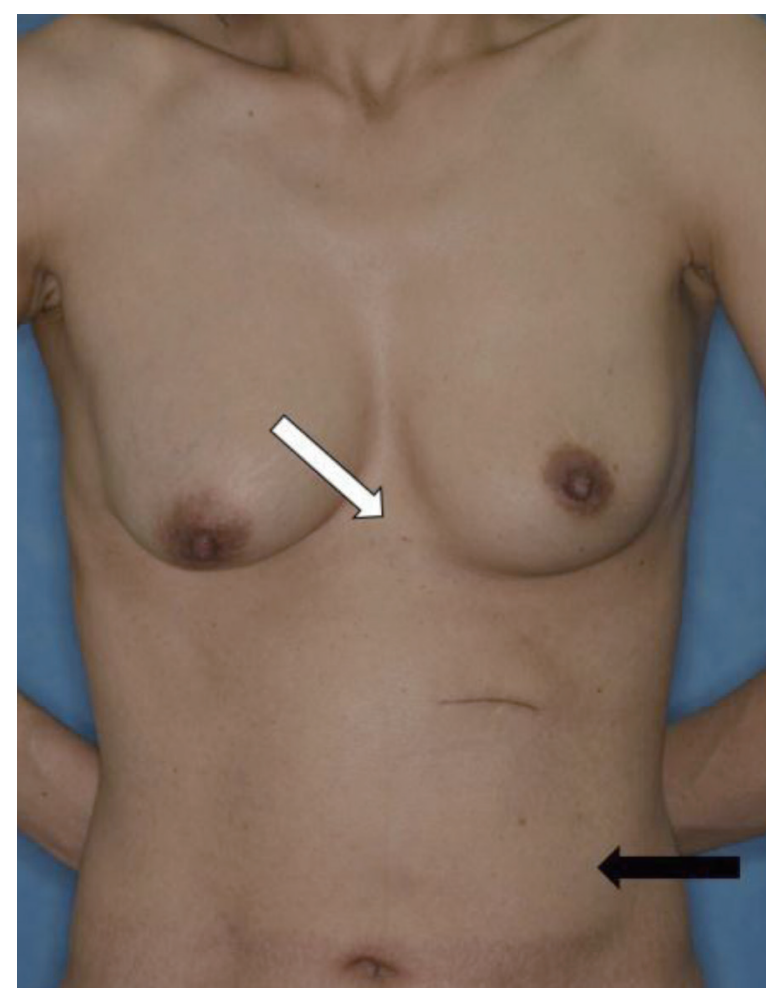

Fig. 1. The patient had masses in the sternal (white arrow) and upper-left abdominal (black arrow) areas.

gins (Fig. 1). Ultrasonography was performed for an initial evaluation. Prior bilateral mammoplasty was revealed on ultrasonography. We noted a large amount of fluid between the envelope and fibrous capsule in the left breast with no evidence of a solid mass in either breast. A hypoechoic lesion of size $3.9 \mathrm{~cm} \times 1.0 \mathrm{~cm} \times 3.4 \mathrm{~cm}$ was found in the sternal area. It was suggestive of an extracapsular rupture of the left breast and formation of a lipoma in the sterna area (Fig. 2). After 1 month, aspiration was performed in both areas. There was thick, bloody discharge from the sternal area. No discharge was noted in the upper-left quadrant of the abdomen. We noted a small mass near the previous mammoplasty scar. We recommended a surgical approach for the masses and the patient provided consent. For further evaluation, computed tomography (CT) was performed before surgery. Material was noted from the left breast implant. A soft tissue lesion appeared to have the same density as the material present on the xiphoid process along the midline subcutaneous layer. Another lesion with a low density was noted on the anterior wall of the upper-left abdomen. The upper part of the lesion was located between the intercostal and external oblique muscles, and the lower part was in the subcutaneous fat layer of the rectus abdominus and oblique muscles, suggestive of leakage from the left breast implant. The CT impression was intraabdominal herniation of the breast implant (Fig. 3). A pathological study of the aspirated material from the surgery was negative for malignant cells. The smear was predominantly composed of his-

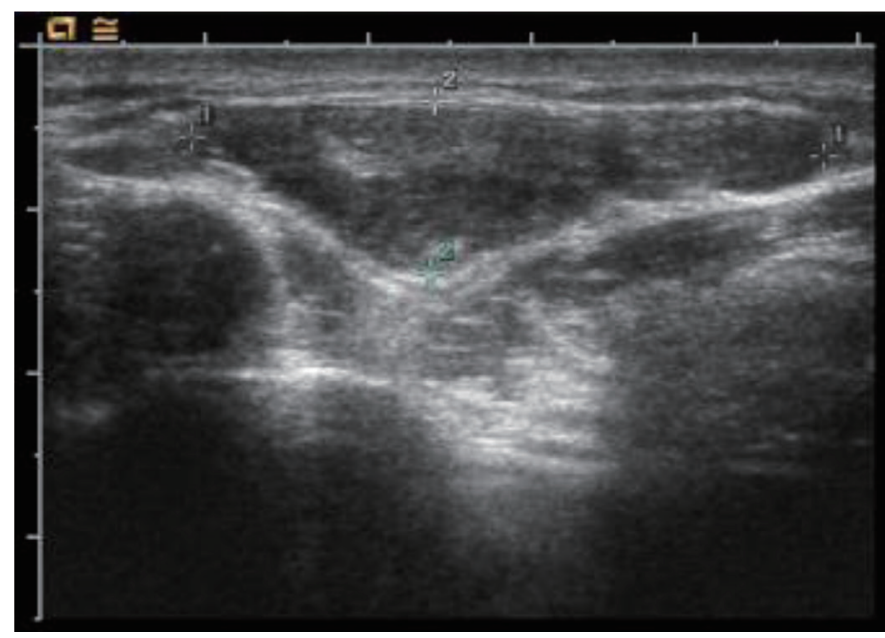

Fig. 2. Ultrasonography revealed a ruptured breast implant and mass in the sternal area.

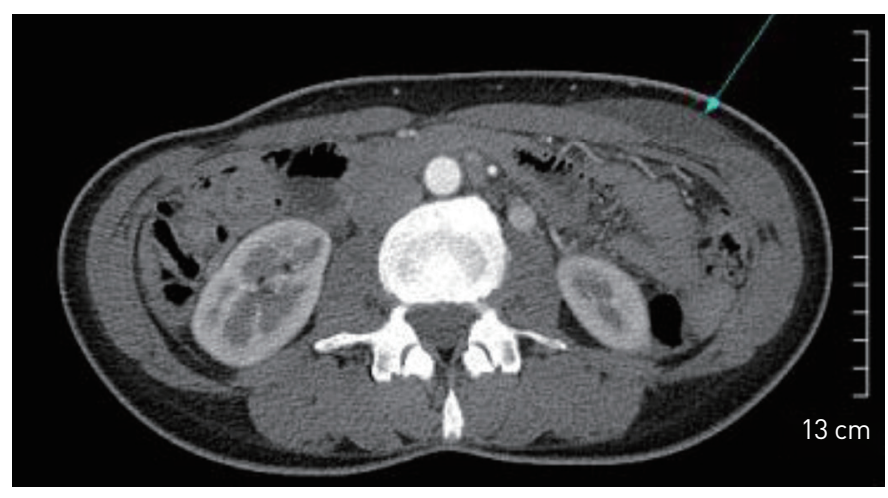

Fig. 3. Enhanced CT revealed a ruptured breast implant in the left breast and a mass in the upper-left abdomen (arrow).

tiocytes lacking atypical lymphoid cells.

During the surgery, the ruptured breast implant $(205 \mathrm{cc}$ ) was located in the submuscular layer of the left breast; an intact implant $(185 \mathrm{cc})$ was located in the right breast. There was yellowish discharge from the left implant. Using the previous mammoplasty incision, a gelatinous, bloody fluid similar to that obtained from the aspirate was noted (Fig. 4). We aspirated all fluid with suction, including the lesion in the upper quadrant of the abdomen through the old incision (Fig. 5). Eight days after surgery, there were no palpable lesions in either the sternal or upper-left abdominal areas.

\section{DISCUSSION}

Migration of material from ruptured breast implants is a rare event. It is associated with extravasation of gel into the breast parenchyma, axillary lymph nodes, chest wall muscles, upper arm, or even the abdominal wall and inguinal areas [6]. The mechanism of silicone migration is not fully understood. Silicone is suggested to mi- 


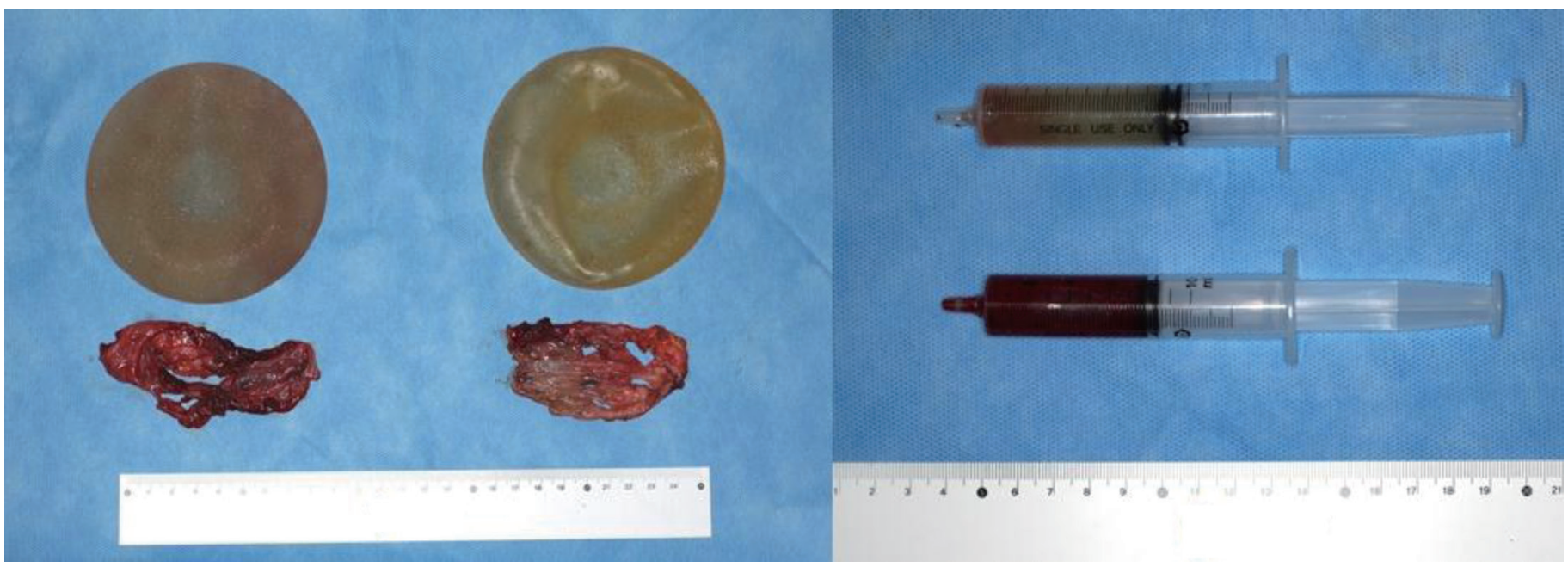

Fig. 4. Breast implant and sternal mass removal, capsulectomy, and suction of the mass in abdomen were performed.

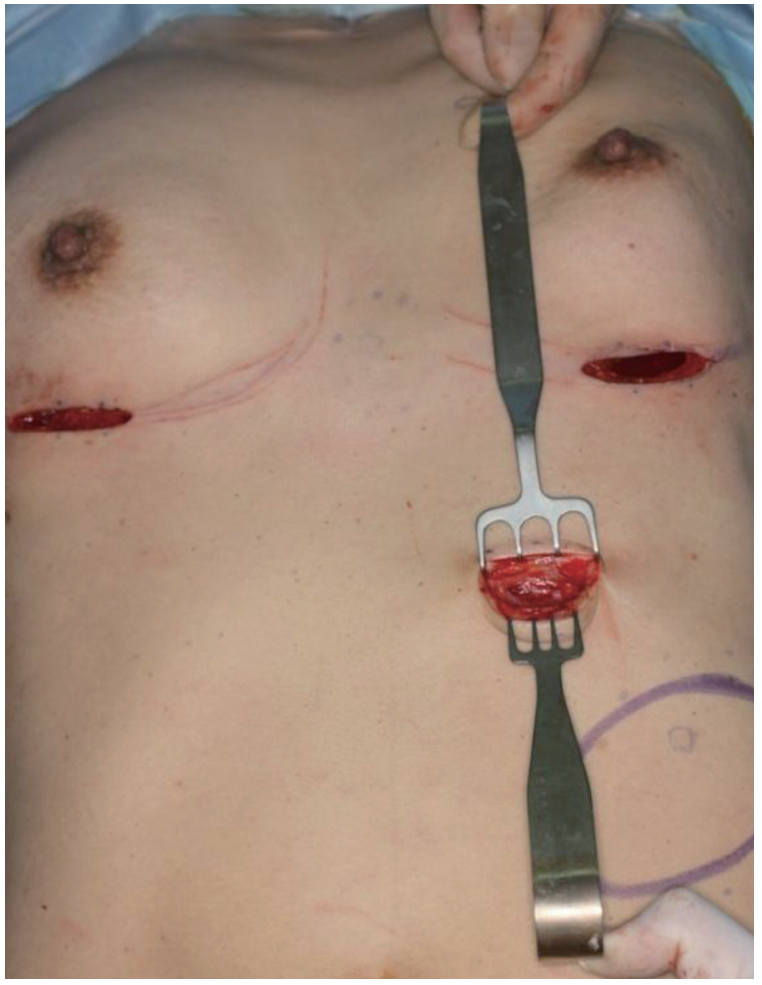

Fig. 5. Removal of breast implants and capsule (left), and aspirated fluid from the left breast and abdomen.

grate through blood, lymphatic vessels, and sites of trauma or surgery. It is rare to report the intra-abdominal migration of material from a ruptured breast implant continuously from the chest to the abdomen. We found just one case of gel migrating into the pleural cavity after injury to the chest wall, one case of migration into the thoracic cavity as observed by video-assisted thoracoscopic surgery for a right middle lobe pulmonary carcinoid, and one case of mi- gration into both legs [7-10].

Imaging protocols for patients with breast implants depend on many factors. If a patient is referred for an evaluation of a possible implant rupture and explantation is being considered, mammography, ultrasonography, or magnetic resonance imaging (MRI) may be required. We did not evaluate the patient by mammography because it has a limited ability to detect silicone rupture and leakage. Dense silicone is not easily penetrated by X-ray energies used for typical mammography [11].

MRI of the breast in the diagnosis of silicone breast implant rupture is widely accepted as the imaging modality of choice for most women. It has been shown to have the highest sensitivity and specificity. However, MRI is the most expensive imaging modality. Therefore, we did not consider it for the initial assessment. Instead, we performed ultrasonography for the patient first, because ultrasonography is relatively inexpensive compared to either MRI or CT. In addition, ultrasonography can detect small amounts of free silicone mixed within the surrounding breast tissues [11].

In addition, we used CT to evaluate the plane of leakage and during the removal of the migrating gel. We suspected that the mass in the patient's abdominal area was from the migration of a ruptured implant instead of a lipoma, because of the similar Hounsfield units detected for the abdominal mass and the silicone material by CT. Culture and cytology from the lesion were performed on the operative field.

Discussing past medical and surgical histories and performing good surgical techniques are important for the diagnosis of a ruptured breast implant. We discussed the common signs of silicone migration and highlighted the importance of awareness among plastic surgeons about this unusual occurrence. If a patient suspects that a breast implant has ruptured, the physician should perform a strict examination of the whole body because of possible migration.

In conclusion, we report a case of silicone migration to the chest 
and abdominal areas from a ruptured breast implant. This case should promote awareness among plastic surgeons about the possibility of this condition in patients with similar symptoms.

\section{REFERENCES}

1. Lake E, Ahmad S, Dobrashian R. The sonographic appearances of breast implant rupture. Clin Radiol 2013;68:851-8.

2. Gabriel SE, Woods JE, O'Fallon WM, et al. Complications leading to surgery after breast implantation. N Engl J Med 1997;336:677-82.

3. Helyar V, Burke C, McWilliams S. The ruptured PIP breast implant. Clin Radiol 2013;68:845-50.

4. Hirsch EM, Seth AK, Fine NA. Capsular contracture after breast reconstruction: A modified classification system incorporating the effects of radiation. Plast Reconstr Surg 2012;129:870e-1e.

5. Handel N, Garcia ME, Wixtrom R. Breast implant rupture: Causes, incidence, clinical impact, and management. Plast Reconstr Surg 2013; 132:1128-37.
6. Sagi L, Baum S, Lyakhovitsky A, et al. Silicone breast implant rupture presenting as bilateral leg nodules. Clin Exp Dermatol 2009;34:e99101.

7. Kim H, Heo C, Baek R, et al. Breast implant migration into pleural cavity. J Plast Reconstr Aesthet Surg 2009;62:e89-90.

8. Mudo ML, Amantea AV, Joaquim AF, et al. Distal migration of ventriculoperitoneal shunting catheter under silicon breast implant. Arq Neuropsiquiatr 2009;67:697-8.

9. Namnoum JD, Largent J, Kaplan HM, et al. Primary breast augmentation clinical trial outcomes stratified by surgical incision, anatomical placement and implant device type. J Plast Reconstr Aesthet Surg 2013; 66:1165-72.

10. Sykes JB, Rosella PA. Intrathoracic migration of a silicone breast implant 5 months after video-assisted thoracoscopic surgery. J Comput Assist Tomogr 2012;36:306-7.

11. David P., Stephanie M., Kathryn L. The diagnosis of silicone breast implant rupture. Plast Reconstr Surg 2007;120:49S-61S. 